\title{
Les variations des glaciers suisses en 1994/95 \\ Rapport préliminaire
}

\begin{abstract}
par Markus Aellen
Laboratoires de recherches hydrauliques, hydrologiques et glaciologiques (VAW) de l'Ecole polytechnique fédérale à Zurich (EPFZ) et Commission des glaciers de l'Académie suisse des sciences naturelles (ASSN).
\end{abstract}

Les levés annuels sur l'état actuel et sur les variations des glaciers des Alpes suisses se fondent essentiellement sur deux projets d'observations à long terme :

- dans le cadre du projet dirigé par la Commission des glaciers et destiné à saisir les variations en longueur, le 116 ème exercice comportait en automne 1995 un nombre total de 112 fronts glaciaires. Les résultats sont reportés dans le tableau 1 et résumés dans le tableau 2. La statistique sommaire de la figure $I$ passe en revue les résultats principaux de la série des 11 '́6 années observées à partir de 1880 :

- dans le cadre du projet dirigé par les VAW et destiné à saisir les variations de masse et du mouvement glaciaire, le bilan de masse a été établi pour trois glaciers et des mesures ponctuelles ont été poursuivies sur six glaciers. Les résultats des bilans établis pour ces trois dernières années sont résumés dans le tableau 3. L'évolution saisonnière du régime hydrologique du bassin versant de la Massa (surface totale $195 \mathrm{~km}^{2}$, englacée sur 2/3 par les glaciers d'Aletsch) est représentée dans la figure 2 par les variations journalières des réserves en eau durant les années hydrologiques 1993/95 et 1994/95, cumulées du ler octobe au 30 septembre et comparées aux données statistiques de la période 1931/1991 (valeur moyenne, écart-type et valeurs extrêmes calculées à l'échelle journalière sur les 60 années hydrologiques).

Les résultats de ces deux dernières années se font remarquer par les bilans de masse modérément déficitaires, en 1994, malgré les chaleurs excessives de l'été, et plus ou moins équilibrés, en 1995, après une saison hivernale très pluvieuse et une saison estivale très chaude mais très courte. Cependant, ces résultats ont été sensiblement réduits après la période du bilan, vu que la fonte durant le mois d'octobre (non comprise dans le bilan) a été bien plus importante que l'accumulation de neige fraiche durant le mois de septembre (comprise dans le bilan). En revanche, la diminution de la longueur des glaciers s'est poursuivie avec une intensité qui dépasse nettement la moyenne $(-7.1 \mathrm{~m} / \mathrm{an})$ des 116 années observées depuis 1880. Bien qu'elle vienne de s'atténuer notablement par rapport aux années précédentes et malgré les bilans de masse peu déficitaires, la tendance à la décrue des glaciers des Alpes suisses est rigoureusement persistante.

LA HOUILLE BLANCHE/N ${ }^{\circ}$ 5-1996 
Tableau 1. - Variations de longueur des glaciers suisses, en 1993/94 et 1994/95.

\begin{tabular}{|c|c|c|c|c|c|c|c|c|c|c|c|}
\hline No. & Glacier & $\begin{array}{l}\text { Variation } \\
\text { 1993/94 }\end{array}$ & $\begin{array}{l}n(\text { en m) } \\
1994 / 95\end{array}$ & No. & Glacier & $\begin{array}{l}\text { Variation } \\
\text { 1993/94 }\end{array}$ & $\begin{array}{l}(\text { en m) } \\
1994 / 95\end{array}$ & No. & Glacier & $\begin{array}{l}\text { Variation } \\
1993 / 94\end{array}$ & $\begin{array}{l}\text { (en m) } \\
1994 / 95\end{array}$ \\
\hline 1 & Rhône & .7 .2 & $-x$ & 39 & Valsorey & -25 & -43 & 73 & Hüfi & -32.62 & 32.6 \\
\hline 2 & Mutt & -0.42 & $\mathrm{n}$ & 40 & Tseudet & -10.4 & -27 & 74 & Griess (Uri) & -17.92 & +0.3 \\
\hline 3 & Gries (Aegina) & -16.82 & $-x$ & 41 & Boveyre & -12.72 & $-x$ & 75 & Fimalpeli & -8.42 & -5 \\
\hline 4 & Fiescher & -20.8 & -17 & 42 & Saleina & -25.7 & -17.7 & 76 & Griessen (Obwa & ald) -4.5 & st \\
\hline 5 & Grosser Aletsch & -36.5 & $-x$ & 108 & Omy & $\mathrm{n}$ & $-x$ & 77 & Biferten & -38.63 & -7 \\
\hline 106 & Mittelaletsch & $-x^{2}$ & $-x$ & 43 & Trient & -57.52 & -40 & 78 & Limmern & -5.82 & -1.7 \\
\hline 6 & Oberaletsch & $-x^{4}$ & $\mathrm{n}$ & 44 & Paneyrosse & -3.82 & +5.8 & 114 & Plattalva & -82 & -3.6 \\
\hline 7 & Kaltwasser & -8.42 & -4.1 & 45 & Gr. Plan Névé & +42 & +5.9 & 79 & Sulz & $x^{2}$ & $-22^{3}$ \\
\hline 8 & Tälliboden & $\mathrm{n}$ & $+x$ & 46 & Martinets & $n$ & $\mathrm{n}$ & 80 & Glärnisch & -9.2 & -34.5 \\
\hline 9 & Ofental & $\mathrm{n}$ & $+x$ & $\because 47$ & Sex Rouge & ?st & st & 81 & Pizol & $-14^{2}$ & sn \\
\hline 10 & Schwarzberg & $-x^{2}$ & $-x$ & 48 & Prapio & -0 & st & 82 & Lavaz & $?$ & $+x$ \\
\hline 11 & Allalin & -11 & $-x$ & 49 & Pierredar & $-x$ & $-x$ & 83 & Punteglias & $-38^{3}$ & -22 \\
\hline 12 & Kessjen & $-x^{2}$ & $+x$ & 50 & Oberaar & -20.4 & & 84 & Lenta & -11.22 & -11 \\
\hline 13 & Fee (Nord) & -55.22 & -37.5 & 51 & Unteraar & -42.8 & & 85 & Vorab & -21.82 & sn \\
\hline 14 & Gorner & -34 & -10 & $\$ 2$ & Gauli & -30 & -12 & 86 & Paradies & $-10.2+$ & +12.1 \\
\hline 15 & Zmutt & +0.3 & st & 53 & Stein & -15 & -12 & 87 & Suretta & +70 & -13.5 \\
\hline 16 & Findelen & $-x^{2}$ & $-x$ & 54 & Steinlimmi & -18 & -3 & 115 & Scaletta & $\mathrm{n}$ & $\mathrm{n}$ \\
\hline 107 & Bis & $-x^{2}$ & $-x$ & 55 & Trift (Gadmen) - & $-x^{2}$ & $-x$ & 88 & Porchabella & -152 & -5.5 \\
\hline 17 & Ried & $-x$ & & 56 & Rosenlaui & $-x$ & $-x$ & 89 & Verstankla & -302 & -3 \\
\hline 18 & Lang & -0.5 & -8 & 57 & Grindelwald sup. - & .62 & -7 & 90 & Silvretta & -6.2 & $-x$ \\
\hline 19 & Tourtemagne & -11.1 & +1.5 & 58 & Grindelwald inf. & st & $-x$ & 91 & Sardona & $-15.72+$ & +27.2 \\
\hline 20 & Brunegg & -8.1 & -5.4 & 59 & Eiger & -12.8 & -8 & 92 & Roseg & -29.9 & -84.4 \\
\hline 21 & Bella Tola & -9 & -39.4 & 60 & Tschingel & -5.4 & -0.2 & 93 & Tschierva & -44.7 & -24.4 \\
\hline 22 & Zinal & -4 & -6 & 61 & Gamchi & -9.4 & -1.2 & 94 & Morteratsch & -12 & -24.1 \\
\hline 23 & Moming & -32 & -16 & 109 & Alpelli (Kander) & -3.2 & -3.4 & 95 & Calderas & -3.6 & -6.9 \\
\hline 24 & Moiry & -0.8 & +0.1 & 110 & Lötschberg & $-x$ & $\mathrm{n}$ & 96 & Tiatscha & $-1.2 \mathrm{ca}$. & st \\
\hline 25 & Ferpecle & -23 & -12.3 & 62 & Schwarz & -43.3 & -1 & 97 & Sesvenna & -8.7 & -1.5 \\
\hline 26 & Mont Miné & -58.4 & -18 & 63 & Lämmem & -5.8 & -4.5 & 98 & Lischana & -5.1 & -6.2 \\
\hline 27 & Arolla (bas) & -6.4 & -11 & 64 & Blümlisalp & -7.1 & -3.3 & 99 & Cambrena & -172 & -9 \\
\hline 28 & Tsidjiore Nouve & +14 & +6 & 111 & Ammerten & -3.9 & -2.1 & 100 & Palü & -8.5 & -7.8 \\
\hline 29 & Cheillon & +112 & -6.7 & 65 & Räızli & 0 & -16 & 101 & Paradisino & -3 & -0.2 \\
\hline 30 & En Darrey & -652 & -2 & 112 & Dungel & $\mathrm{n}$ & $\mathrm{n}$ & 102 & Forno & -24 & -20.7 \\
\hline 31 & Grand Désert & -11.22 & -3.8 & 113 & Gelten & $n$ & $\mathrm{n}$ & 116 & Albigna & $\mathrm{n}$ & $n$ \\
\hline 32 & Mt.FortTortin & +5.22 & +22.5 & 66 & Tiefen & -20.42 & +2.5 & 120 & Como & -16.32 & +1 \\
\hline 33 & Tsanfleuron & -2 & -17.5 & 67 & St. Anna & -7.22 & -0.9 & 117 & Valleggia & -1.5 & -7 \\
\hline 34 & Otemma & -32.3 & - & 68 & Kehlen & -41.42 & -29.3 & 118 & Val Torta & +9 & -4.6 \\
\hline 35 & Mont Durand & +8 & +8.2 & 69 & Rotfirn & -92 & -11.4 & 103 & Bresciana & -8 & -13.5 \\
\hline 36 & Breney & -7.8 & -1.7 & 70 & Danna & .8 .92 & -15.8 & 119 & Cavagnoli & -11.92 & -0.2 \\
\hline 37 & Giétro & $-x$ & $-x$ & 71 & Wallenbur & -12.82 & -2.8 & 104 & Basodino & $?+6.0^{3}$ & -24.5 \\
\hline 38 & Corbassière & $\mathrm{n}$ & $-x$ & 72 & Brunni & $-x^{3}$ & $\mathrm{n}$ & $\begin{array}{r}\mathrm{Cr} \\
105\end{array}$ & $\begin{array}{l}\text { Croslina } \\
\text { Rossboden }\end{array}$ & $\begin{array}{c}0 \\
+3.2\end{array}$ & $\begin{array}{l}-7.6 \\
-8.5\end{array}$ \\
\hline \multicolumn{12}{|c|}{ Abréviations: } \\
\hline \multirow{3}{*}{\multicolumn{4}{|c|}{$\begin{array}{l}+ \text { en crue } \\
\text { st stationnaire } \\
\text { - en décrue }\end{array}$}} & \multirow{3}{*}{\multicolumn{4}{|c|}{$\begin{aligned}+7^{2} & \text { avance de } 7 \mathrm{~m} \text { en deux } \\
\times & \text { valeur inconnue } \\
\mathrm{ca} & \text { valeur approximative }\end{aligned}$}} & ? & \multirow{3}{*}{\multicolumn{3}{|c|}{$\begin{array}{l}\text { résultat incertain } \\
\text { sous la neige } \\
\text { non observé }\end{array}$}} \\
\hline & & & & & & & & sn & & & \\
\hline & & & & & & & & $\mathrm{n}$ & & & \\
\hline
\end{tabular}


a) Nombre des glaciers observés (N) et nombre de valeurs comprises dans la moyenne (M)

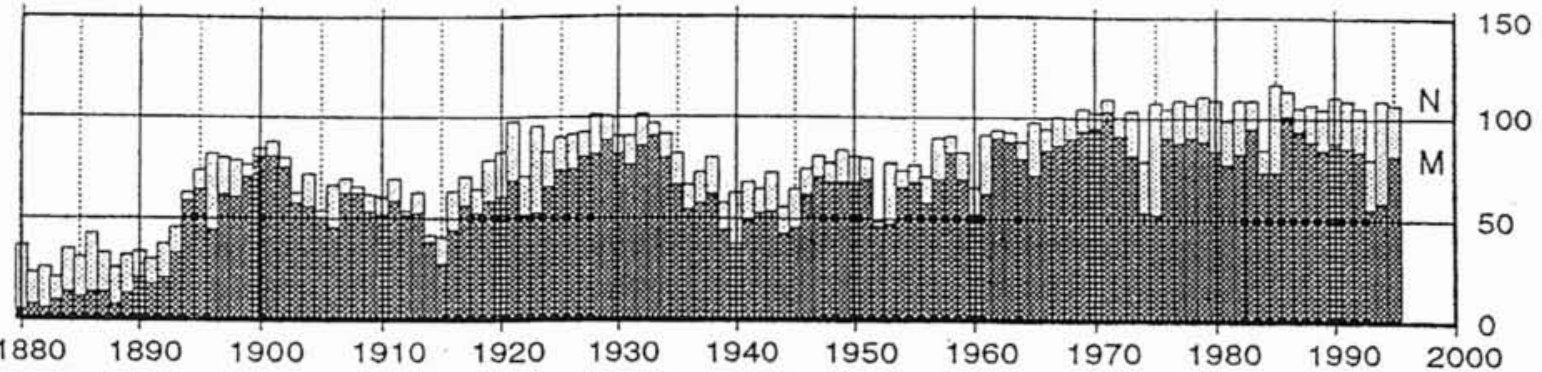

b) Pourcentages des glaciers en crue et des glaciers en décrue

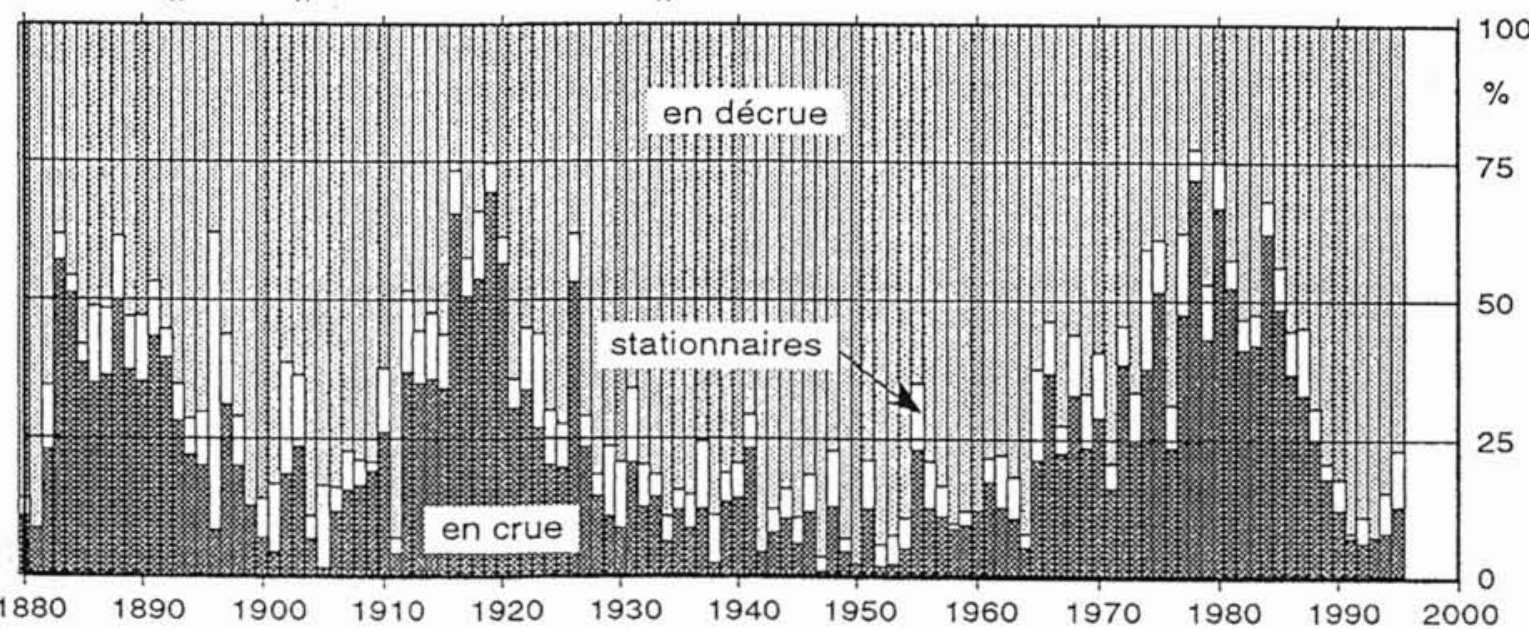

c) Variation moyenne de la longueur

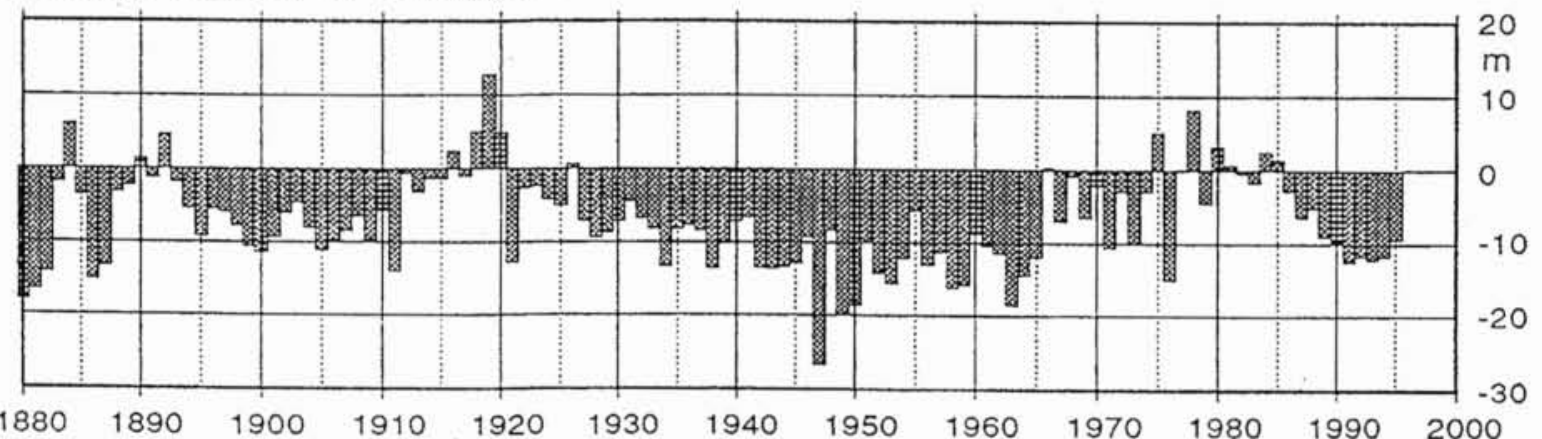

c) Variations cumulées de la longueur des glaciers du Rhône et du Trient comparées à la moyenne

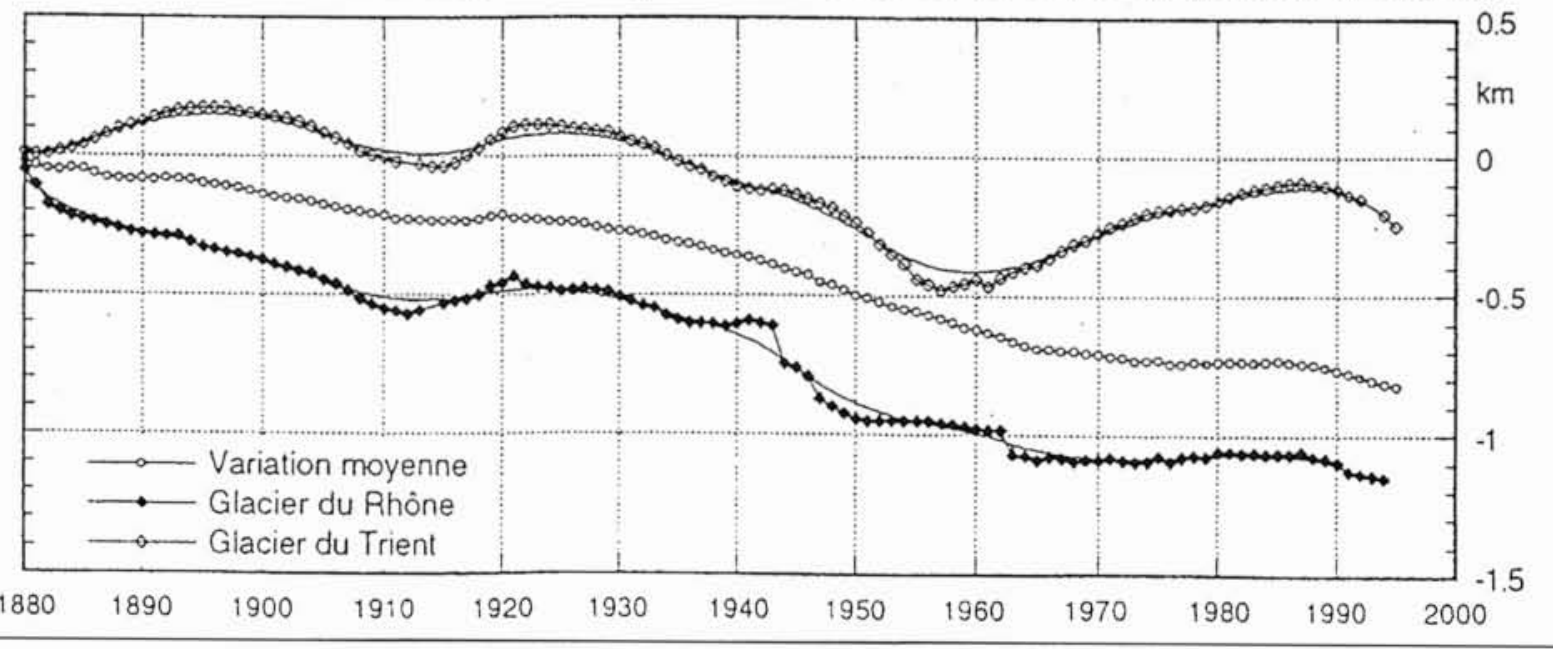

1. Variations de la longueur des glaciers dans les Alpes suisses de 1880 à 1995. 
Tableau 2. - Variations de longueur des glaciers suisses, en 1993/94 et 1994/95. Récapitulation

\begin{tabular}{|c|c|c|c|c|c|c|c|c|c|}
\hline \multirow[t]{2}{*}{ Bassins versants } & \multirow[t]{2}{*}{$\begin{array}{l}\text { Nombre de glaciers } \\
\text { Réseau }\end{array}$} & \multicolumn{2}{|c|}{ classés } & \multicolumn{2}{|c|}{ en crue } & \multicolumn{2}{|c|}{ stationnaires } & \multicolumn{2}{|c|}{ en décrue. } \\
\hline & & $93 / 94$ & $94 / 95$ & $93 / 94$ & $94 / 95$ & $93 / 94$ & $94 / 95$ & $93 / 94$ & $94 / 95$ \\
\hline Rhône & 52 & 46 & 48 & 5 & 9 & 5 & 4 & 36 & 35 \\
\hline $\begin{array}{l}\text { Aar } \\
\text { Reuss }+ \text { limmat }\end{array}$ & 21 & 19 & 16 & & 1 & 2 & 1 & 17 & 15 \\
\hline $\begin{array}{l}\text { Reuss + Limmat } \\
\text { Rhin + Inn }\end{array}$ & 18 & $\begin{array}{l}16 \\
16\end{array}$ & 16 & 1 & 3 & & 1 & $\begin{array}{l}16 \\
15\end{array}$ & $\begin{array}{l}11 \\
12\end{array}$ \\
\hline Adda + Tessin & 13 & 12 & 12 & 3 & 1 & 1 & 2 & 8. & 9 \\
\hline Total & 121 & 1091 & 1071 & $9^{2}$ & $14^{2}$ & $8^{3}$ & $11^{3}$ & $92^{4}$ & $82^{4}$ \\
\hline Pourcentages & & 100 & & 8 & 13 & 7 & 10 & 85 & 77 \\
\hline $\begin{array}{l}\text { Variation moyenne } \\
\text { Nombre de valeurs }\end{array}$ & & $\begin{array}{r}-11.8 \\
59^{5}\end{array}$ & $\begin{array}{l}-9.4 \\
845\end{array}$ & & & & & & \\
\hline
\end{tabular}

Classification : les classes comprennent les glaciers cités ci-après par leur numéro du tableau 1 :

- en 1993/94:

1) non observés: 89384679108112113115116 : résultat incertain : 4782 .

2) en crue: 282932354587104105118 .

3) stationnaires : 2 is $1824485865 \mathrm{Cr}$

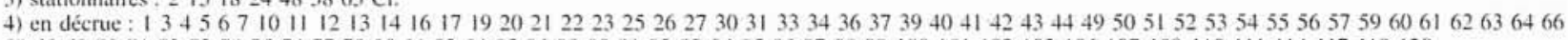
6768697071727374757677788081838485868890919293949596979899100101102103106107109110111114117119120.

5) omis: $2367101213161729303132374143+4-4549505556666768697071727374757778818384858889919699104106107110114$ 119120.

- en 1994/95:

1) non observés : $264672110112113115116:$ résultat incertain : 8185 ; résúltat à confirmer: 175051 .

2) en crue : 891219283235444566828691120 .

3) stationnaires: 152447486067747696101119

4) en décrue : 1345710111314161820212223252627293031333436373839404142434952535455565758596162636465686970 $7173757778798083848788899092939495979899100102103104105106107108109111114117118 \mathrm{Cr}$.

5) omis: I 3589101112151637384149555658798290106107108.

Tableau 3. - Bilan de masse de quelques glaciers, en 1992/93 - 1994/95.

\begin{tabular}{|lcccc|}
\hline Glacier & Année de bilan & $\begin{array}{c}\text { Surface } \\
\mathrm{km}^{2}\end{array}$ & $\begin{array}{c}\text { Bilan spécifique } \\
\mathrm{g} / \mathrm{cm}^{2} \text { (cm w.e.) }\end{array}$ & $\begin{array}{c}\text { Ligne d'équilibre } \\
\mathrm{m} \text { s.m. }\end{array}$ \\
\hline Gries & $1992 / 93$ & 6.31 & -23 & 2872 \\
& $1993 / 94$ & 6.31 & -75 & 3018 \\
Aletsch & $1994 / 95$ & 6.31 & -7 & 2848 \\
& $1992 / 93$ & 127.24 & $-45^{*}$ & $2910^{*}$ \\
Silvretta & $1993 / 94$ & 127.15 & $-28^{*}$ & $2980^{*}$ \\
& $1994 / 95$ & 127.10 & -5 & 2778 \\
& $1992 / 93$ & 3.15 & -47 & 2852 \\
\hline
\end{tabular}

s) valeurs provisoires

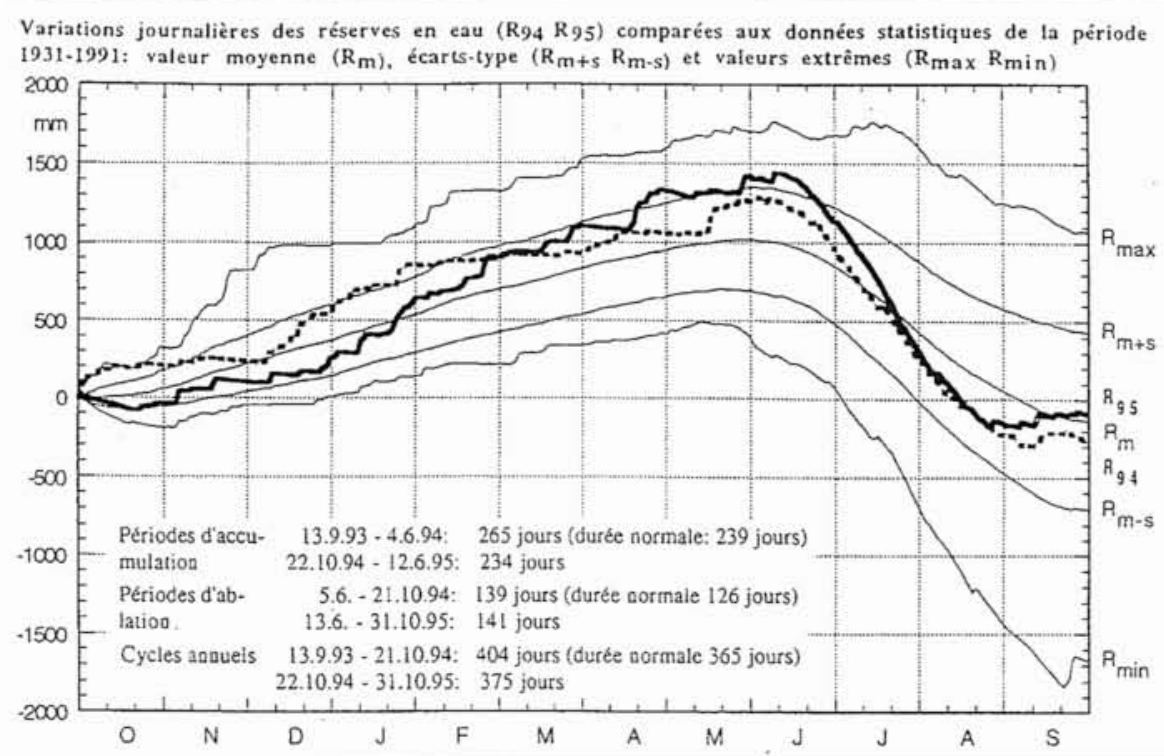

2. Bassin versant de la Massa (glaciers d'Aletoch). Bilans hydrologiques 1993/94 et 1994/95. 